\title{
ON THE EXISTENCE OF NONSIMPLE REAL EIGENVALUES FOR GENERAL STURM-LIOUVILLE PROBLEMS ${ }^{1}$
}

\author{
A. B. MINGARELLI
}

\begin{abstract}
The Sturm-Liouville eigenvalue problem $-y^{\prime \prime}+q(t) y=\lambda r(t) y, t \in$ $[a, b]$, where $y$ is required to satisfy a pair of homogeneous separated boundary conditions at $t=a, t=b$ is considered when no sign restrictions are imposed upon the coefficients $q, r$. It will be shown that the general eigenvalue problem above can admit at most finitely many nonsimple real eigenvalues (in some cases none at all). Moreover, we will show by means of an example that nonsimple real eigenvalues may occur in the case when each of $q$ and $r$ changes sign in $(a, b)$ and under Dirichlet boundary conditions
\end{abstract}

1. Let $q, r:[a, b] \rightarrow \mathbf{R}$ and $q, r \in L(a, b)$. We will always assume $\int_{a}^{b}|r|>0$. The problem under consideration is the general Sturm-Liouville eigenvalue problem

$$
-y^{\prime \prime}+q(t) y=\lambda r(t) y, \quad a \leqslant t \leqslant b,
$$

where we require $y$ to satisfy

$$
\begin{aligned}
& y(a) \cos \alpha-y^{\prime}(a) \sin \alpha=0, \\
& y(b) \cos \beta+y^{\prime}(b) \sin \beta=0,
\end{aligned}
$$

for $0 \leqslant \alpha, \beta<\pi$. If we let $y(t, \lambda)$ be a nontrivial solution of (1.1) then classical methods give that $y(t, \lambda)$ is an entire function of $\lambda$ for fixed $t$ and, in fact, its order is at most $\frac{1}{2}$ in the general case under consideration, cf. [4].

In [6] R. G. D. Richardson showed that under the assumptions $q, r \in C[a, b]$ the problem (1.1-1.3) admits, in the nondefinite case [6, \$4], a countable number of real eigenvalues; the collection of which is unbounded above and below. The analog of this result for $q, r \in L(a, b)$ and $r$ as above was obtained by Atkinson-Mingarelli [1]. Moreover, it was shown in [5] that under the latter condition on $q, r$ the problem (1.1-1.3) has an at most finite (though possibly empty) set of nonreal eigenvalues (which necessarily occur in complex conjugate pairs).

The question of the simplicity of the real eigenvalues (as zeros of the characteristic equation) is of current interest. We will show below that, apart from an at most finite number of exceptions, all the real eigenvalues are simple. However, we will also give an example illustrating the fact that nonsimple real eigenvalues can exist, even in the case of Dirichlet boundary conditions.

Received by the editors November 29, 1982. The results of this paper were presented at the 64 th Ontario Mathematics Meeting, Carleton University, Ottawa, November 27, 1982.

1980 Mathematics Subject Classification. Primary 34B25.

Key words and phrases. Sturm-Liouville problems, nonsimple eigenvalues.

1 This research is funded by the Natural Sciences and Engineering Research Council of Canada under grant UO 167. 
2. The following result was stated by Richardson [6, p. 294] though not proved. A proof is included for the sake of completeness.

Lemma 1.1. Let $\lambda, y(t, \lambda)$ be an eigenvalue-eigenfunction pair of (1.1-1.3) with $\lambda$ real or nonreal and where we assume, more generally, that $q, r \in L(a, b)$.

Then a necessary and sufficient condition that $\lambda$ be nonsimple is that

$$
\int_{a}^{b}\{y(t, \lambda)\}^{2} r(t) d t=0 .
$$

We leave the proof until the next section. The main result is the following:

THEOREM 1.1. Let $\lambda=0$ not be an eigenvalue of (1.1-1.3). Then for $\lambda>0$ (resp. $\lambda<0$ ) there is an at most finite (though possibly empty) set of distinct eigenvalues with the property that corresponding eigenfunctions $y(t, \lambda)$ satisfy

$$
\int_{a}^{b}\{y(t, \lambda)\}^{2} r(t) d t \leqslant 0 \quad(\text { resp. } \geqslant 0) .
$$

REMARK 1. The assumption that $\lambda=0$ is not an eigenvalue is not restrictive as the translation $\lambda \rightarrow \lambda+c$ where $c \in \mathbf{R}, c \neq 0$ is sufficiently small, transforms (1.1) into a problem of the same form wherein $\lambda=0$ is not an eigenvalue.

2. A combination of Lemma 1.1 and Theorem 1.1 now yields the existence of an at most finite collection of nonsimple real eigenvalues. A by-product of the proof of Theorem 1.1 is

COROLlaRY 1.1. The total number of distinct real eigenvalues of $(1.1-1.3)$ which are nonsimple does not exceed twice the total number of negative eigenvalues of the corresponding problem

$$
-y^{\prime \prime}+q(t) y=\lambda y, \quad a \leqslant t \leqslant b,
$$

where $y$ is required to satisfy (1.2-1.3).

Remark 3. Let $r(t)=A$ for $t \in[0,1) ; r(t)=-A$ for $t \in[1,2]$ where $A \neq 0$ is a real constant. Define $q$ by $q(t)=r(t)-9 \pi^{2} / 4$, for $t \in[0,2]$. Let $\alpha=0=\beta$ and $a=0, b=2$. Then it is readily seen that $\lambda=1$ is an eigenvalue of $(1.1-1.3)$ with a corresponding eigenfunction $y(t, \lambda)=\sin (3 \pi t / 2), t \in[0,2]$, satisfying (2.1). Hence $\lambda=1$ is nonsimple on account of Lemma 1.1. Other examples may be constructed similarly in the case of more general boundary conditions.

4. Finally, we note that strict inequality may occur in (2.2). To see this let $A=1$ and define $q, r$ as in Remark 3 above except that now we require $r(t)=+1$ on $[0,1)$ and $r(t)=-1$ on $[1,4]$. Let $\alpha=0=\beta$ and $a=0, b=4$. Once again, $\lambda=1$ is an eigenvalue with $y(t, \lambda)=\sin (3 \pi t / 2)$ as a corresponding eigenfunction for which $\int_{0}^{4} r(t) y^{2}(t, \lambda) d t<0$. It is now readily seen that there may exist negative eigenvalues whose eigenfunctions have the property $\int_{a}^{b} r(t) y^{2}(t, \lambda) d t>0$.

3. Proof of Lemma 1.1. Let $y(t, \lambda) \neq 0$ be a solution which satisfies (1.2). The characteristic equation which defines the eigenvalues is then

$$
F(\lambda) \equiv \cos \beta y(b, \lambda)+\sin \beta y^{\prime}(b, \lambda)=0 .
$$


Following the method described in $[3, \S 10.72]$ we readily obtain the identity

$$
(\cos \beta)\left(\lambda-\lambda_{i}\right) \int_{a}^{b} y(t, \lambda) y\left(t, \lambda_{i}\right) r(t) d t=y^{\prime}\left(b, \lambda_{i}\right) F(\lambda)
$$

(if $\cos \beta \neq 0$ ). Since $F\left(\lambda_{i}\right)=0$ and $F$ is entire, we immediately see that (upon letting $\left.\lambda \rightarrow \lambda_{i}\right)$

$$
\cos \beta \int_{a}^{b}\left\{y\left(t, \lambda_{i}\right)\right\}^{2} r(t) d t=y^{\prime}\left(b, \lambda_{i}\right) \cdot F^{\prime}\left(\lambda_{i}\right) .
$$

Hence, $F^{\prime}\left(\lambda_{i}\right)=0$ if and only if there holds $(2.1)\left(\right.$ as $y^{\prime}\left(b, \lambda_{i}\right) \neq 0$ if $\left.\cos \beta \neq 0\right)$. Minor changes in the proof apply to the case $\cos \beta=0$, and so will be omitted.

Proof of Theorem 1.1. It suffices to prove the theorem for $\lambda>0$ as the other case follows from similar considerations. Thus let $\mu_{1}<\mu_{2}<\cdots<\mu_{n}<0$ denote the $N$ negative eigenvalues of the problem $(2.3-1.2-1.3)$ and $\psi_{j}, j=1, \ldots, N$ denote $N$ corresponding eigenfunctions. If possible, assume there exists $M>N$ distinct real eigenvalues of (1.1-1.2-1.3), each of which has a real eigenfunction satisyfing (2.2). Let us fix such a set of eigenfunctions and label them $\phi_{1}, \phi_{2}, \ldots, \phi_{M}$, the order being of no importance here. Then each $\phi_{i}$ satisfies (2.1) and a Sturmian argument shows that

$$
\int_{a}^{b} \phi_{i}(t) \phi_{j}(t) r(t) d t=0
$$

for $i \neq j, 1 \leqslant i, j \leqslant M$. Now let $\psi=\sum_{j=1}^{M} c_{j} \phi_{j}$ be chosen so that $\psi$ is $L^{2}$-orthogonal to each $\psi_{i}, i=1,2, \ldots, N$. Since $M>N$, a nontrivial collection of such $c_{j}$ 's may always be found. Moreover, $\psi \neq 0$ as the $\phi_{i}$ are linearly independent functions over C (being eigenfunctions corresponding to distinct eigenvalues). Fixing such a set of $c_{j}$ 's, it is readily verified that $\psi$ satisfies $(1.2-1.3)$ and, in fact, $\psi(t)$ satisfies $(2.2)$ which can be easily seen once use is made of (3.2).

Multiplying (1.1) by $y(t, \lambda)$, integrating the result over $[a, b]$ and making use of (1.2-1.3), one finds that for $y=\phi_{i}$

$$
\begin{aligned}
\left(Q \phi_{i}, \phi_{i}\right) & \equiv \int_{a}^{b}\left\{\phi_{i}^{\prime 2}+q \phi_{i}^{2}\right\} d t+\cot \alpha \cdot \phi_{i}^{2}(a)+\cot \beta \cdot \phi_{i}^{2}(b) \\
& =\lambda_{i} \int_{a}^{b} \phi_{i}^{2} r d t \leqslant 0, \quad i=1,2, \ldots, M,
\end{aligned}
$$

since $\lambda_{i}>0$. Now $(Q \psi, \psi)=\sum_{j=1}^{M} \sum_{i=1}^{M} c_{i} \bar{c}_{j}\left(Q \phi_{i}, \phi_{j}\right)$. Since $\left(Q \phi_{i}, \phi_{j}\right)=0$ for $i \neq j$, we find $(Q \psi, \psi)=\sum_{i=1}^{M}\left|c_{i}\right|^{2}\left(Q \phi_{i}, \phi_{i}\right) \leqslant 0$ by a former result. We have thus found a function $\psi \neq 0$ with $\psi$ being $L^{2}$-orthogonal to $\psi_{i}, i=1,2, \ldots, N$, and such that $(Q \psi, \psi) \leqslant 0$. Now (e.g. [2]),

$$
\mu_{N+1}=\min _{f \neq 0} \frac{(Q f, f)}{(f, f)}
$$

where the minimum is over those $f \in D \equiv\left\{f \in L^{2}(a, b): f, f^{\prime}\right.$ are absolutely continuous on $[a, b]$ and $-f^{\prime \prime}+q(t) f$ in $L^{2}(a, b)$, and $f$ satisfies (1.2-1.3) $\}$ which are orthogonal to each of $\psi_{1}, \psi_{2}, \ldots, \psi_{N}$ in the $L^{2}$-sense. But $\psi$ is such a function-hence 
$\mu_{N+1} \leqslant((Q \psi, \psi) / \psi, \psi) \leqslant 0$. However, since $\lambda=0$ is not an eigenvalue of $(2.3-1.2-$ 1.3), by hypothesis, we must have $\mu_{N+1}>0$. This contradiction implies that the hypothesis $M>N$ is untenable and so $M \leqslant N$. Since $N$ is always finite under the stated assumption on $q$ (by Sturm-Liouville theory) this completes the proof of the theorem and, at the same time, the corollary.

\section{REFERENCES}

1. F. V. Atkinson and A. B. Mingarelli, private correspondence.

2. R. Courant and D. Hilbert, Methods of mathematical physics, Vol. 1, Interscience, New York, 1953.

3. E. Ince, Ordinary differential equations, Dover, New York, 1956.

4. A. B. Mingarelli, Some remarks on the order of an entire function associated with a second order differential equation, (Tribute to F. V. Atkinson, Proc. Sympos. on Differential Operators, Dundee, Scotland, March-April-May 1982), Lecture Notes in Math., Springer-Verlag, Berlin and New York (to appear).

5. Indefinite Sturm-Liouville problems, Lecture Notes in Math., Vol. 964, Springer-Verlag, Berlin and New York, 1983, pp. 519-528.

6. R. G. D. Richardson, Contributions to the study of oscillation properties of the solutions of linear differential equations of the second order, Amer. J. Math. 40 (1918), 283-316.

Department of Mathematics, University of Ottawa, Ottawa, Ontario, Canada KIN 9B4 